MATHEMATICS OF COMPUTATION

Volume 77, Number 262, April 2008, Pages 633-649

S 0025-5718(07)02030-3

Article electronically published on December 12, 2007

\title{
A POSTERIORI ERROR ESTIMATES FOR MAXWELL EQUATIONS
}

\author{
JOACHIM SCHÖBERL
}

\begin{abstract}
Maxwell equations are posed as variational boundary value problems in the function space $H$ (curl) and are discretized by Nédélec finite elements. In Beck et al., 2000, a residual type a posteriori error estimator was proposed and analyzed under certain conditions onto the domain. In the present paper, we prove the reliability of that error estimator on Lipschitz domains. The key is to establish new error estimates for the commuting quasi-interpolation operators recently introduced in J. Schöberl, Commuting quasi-interpolation operators for mixed finite elements. Similar estimates are required for additive Schwarz preconditioning. To incorporate boundary conditions, we establish a new extension result.
\end{abstract}

\section{INTRODUCTION}

Maxwell equations are partial differential equations describing electro-magnetic phenomena. In comparison to other fields, their numerical treatment by finite element methods is relatively new. One reason is that they require the vector valued function space $H$ (curl), which has many consequences for numerical analysis as a whole. A recent monograph is [18].

The key for the numerical analysis for Maxwell equations is most often the de Rham complex [8. It is the basis for the construction of finite elements [19, 20, 31, 14, 1, 26, 32, and the a priori error estimates, preconditioners [16, 3, 28, 22, and eigenvalue problems [6, 7].

The principle of energy-based a posteriori error estimators 30 , 2 is the localization of error contributions. For the residual error estimator, the Clément operator is applied to subtract a global function. By a partition of unity method, the rest can be split into local functions. The same concept is needed for two-level domain decomposition methods. After subtracting a coarse grid function, the remainder can be split into local functions on overlapping sub-domains [29].

Residual based a posteriori error estimators for Maxwell equations were introduced in [4]. In [17], scattering problems were treated. In these papers, proper element and inter-element jump terms have been derived. In [21, 12] the heterogeneous Maxwell equation was addressed. An alternative are hierarchical error estimators 5 or equilibrated residual error estimators [9]. In the present paper, we

Received by the editor May 5, 2005 and, in revised form, July 25, 2006.

2000 Mathematics Subject Classification. Primary 65N30.

Key words and phrases. Clément operator, Maxwell equations, edge elements.

The author acknowledges support from the Johann Radon Institute for Computational and Applied Mathematics (RICAM), Austria, and from the Austrian Science Foundation FWF within project grant Start Y-192, "hp-FEM: Fast Solvers and Adaptivity".

(C)2007 American Mathematical Society Reverts to public domain 28 years from publication 
prove the reliability of residual error estimators on Lipschitz domains. The key is to establish new error estimates for the commuting quasi-interpolation operators introduced recently in [25]. These operators are no projectors. In [27, the operators have been modified to obtain the projection property as well.

Notation: We write $a \preceq b$, when $a \leq c b$, where $c$ is a constant independent of $a$, $b$, the coefficients $\nu$ and $\kappa$ of the equation, and the mesh-size $h$. The constant may depend on the shape of the finite elements. We write $a \succeq b$ for $b \preceq a$, and we write $a \simeq$ for $a \preceq b$ and $b \preceq a$.

The rest of the paper is organized as follows. In Section 2, the variational problem, the error estimator and the main theorem is presented. The commuting quasi-interpolation operators are defined in Section 3, and the new approximation properties are proven in Section 4. Necessary extension results for $H$ (curl) and $H$ (div) are proven in Appendix $\mathrm{A}$

\section{THE RESIDUAL ERROR ESTIMATOR}

Let $\Omega$ be a bounded, polyhedral Lipschitz domain in $\mathbb{R}^{3}$. Its boundary $\Gamma=\partial \Omega$ is decomposed into the Dirichlet part $\Gamma_{D}$ and the Neumann part $\Gamma_{N}$. As usual, define the space $H(\operatorname{curl}, \omega)=\left\{v \in\left[L_{2}(\omega)\right]^{3}: \operatorname{curl} v \in\left[L_{2}(\omega)\right]^{3}\right\}$ for some domain $\omega$, and write $H$ (curl) for $\omega=\Omega$. Let $V:=H_{D}(\operatorname{curl}):=\left\{v \in H(\operatorname{curl}): v_{t}=0\right.$ on $\left.\Gamma_{D}\right\}$. Similarly, we define $H_{D}^{1}=\left\{v \in H^{1}: v=0\right.$ on $\left.\Gamma_{D}\right\}$. We write $v_{t}$ and $v_{n}$ for the tangential and normal traces, respectively.

Several formulations of Maxwell equations lead to the variational problem: find $u \in V$ such that

$$
A(u, v)=f(v) \quad \forall v \in V
$$

with the bilinear-form

$$
A(u, v):=\int_{\Omega} \nu(x) \operatorname{curl} u \operatorname{curl} v d x+\int_{\Omega} \kappa(x) u v d x
$$

and the linear form $f($.$) defined as$

$$
f(v):=\int_{\Omega} j v d x .
$$

The coefficients $\nu(x)$ and $\kappa(x)$ are modified material parameters. In time-stepping methods, $\kappa(x)$ includes the time step $\Delta t$, while in time harmonic formulations, the equation becomes complex-valued with $\kappa(x)=i \omega \sigma-\omega^{2} \varepsilon$, where $\sigma$ and $\varepsilon$ are positive coefficient functions. We assume that the bilinear-form $A(.,$.$) is continuous$ and inf - sup stable with respect to the norm

$$
\|v\|_{V}^{2}:=\nu\|\operatorname{curl} v\|_{L_{2}}^{2}+\kappa\|v\|_{L_{2}}^{2},
$$

where $\nu$ and $\kappa$ are positive constants. The given current density $j \in\left[L_{2}\right]^{3}$ satisfies $\operatorname{div} j=0$ and $j_{n}=0$.

Let the domain $\Omega$ be covered with a shape regular triangulation. We define

$$
\begin{aligned}
\text { the set of vertices } & \mathcal{V}=\left\{V_{i}\right\}, \\
\text { the set of edges } & \mathcal{E}=\left\{E=\left[V_{E_{1}}, V_{E_{2}}\right]\right\}, \\
\text { the set of faces } & \mathcal{F}=\left\{F=\left[V_{F_{1}}, V_{F_{2}}, V_{F_{3}}\right]\right\}, \\
\text { the set of tetrahedra } & \mathcal{T}=\left\{T=\left[V_{T_{1}}, V_{T_{2}}, V_{T_{3}}, V_{T_{4}}\right]\right\} .
\end{aligned}
$$



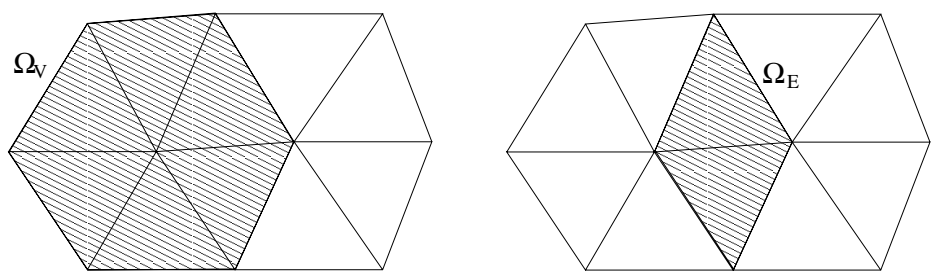

Figure 1. Element patches $\Omega_{V}$ and $\Omega_{E}$
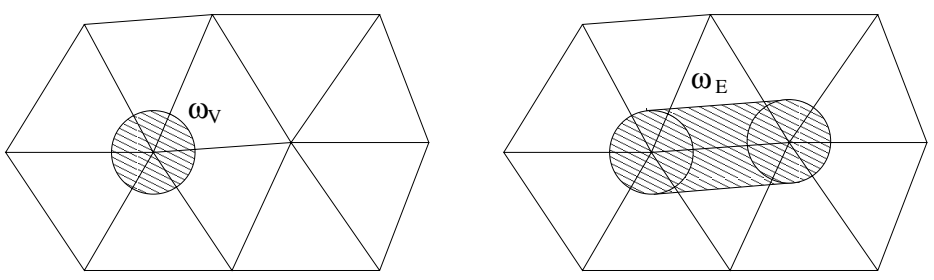

Figure 2. Domains $\omega_{V}$ and $\omega_{E}$

For each edge $E$ we define a unique tangential vector $t_{E}$, and for each face $F$ we define a unique normal vector $n_{F}$. For each edge $E$, face $F$, and element $T$ the local mesh-size $h_{E}, h_{F}$, and $h_{T}$ is defined by the diameter, and for a vertex $V$ the mesh-size $h_{V}$ is defined as $\max _{T: V \in T} h_{T}$. Note that all geometric entities are closed sets. We need several domains associated with the entities of the mesh. First, define the small patches associated with vertices, edges and faces as

$$
\Omega_{V}=\bigcup_{T: V \in T} T, \quad \Omega_{E}=\bigcup_{T: E \subset T} T, \quad \Omega_{F}=\bigcup_{T: F \subset T} T ;
$$

see Figure 1. We will need the influence domains of the interpolation operators. For this, let $\omega_{V} \subset \Omega_{V}$ be a domain with three dimensional measure $\left|\omega_{V}\right| \simeq h_{V}^{3}$. It can be a ball with center $V$, and a radius proportional to the local mesh-size. We assume that $\operatorname{dist}\left\{\omega_{V_{i}}, \omega_{V_{j}}\right\} \succeq\left|V_{i}-V_{j}\right|$. Furthermore, let

$$
\omega_{E}=\left[\omega_{E_{1}}, \omega_{E_{2}}\right], \quad \omega_{F}=\left[\omega_{F_{1}}, \omega_{F_{2}}, \omega_{F_{3}}\right], \quad \omega_{T}=\left[\omega_{T_{1}}, \omega_{T_{2}}, \omega_{T_{3}}, \omega_{T_{4}}\right]
$$

be the convex hulls of the domains associated with the vertices of the edge $E$, the face $F$, and the element $T$; see Figure 2. We assume that $\omega_{E} \subset \bigcup_{V \in E} \Omega_{V}$, $\omega_{F} \subset \bigcup_{V \in F} \Omega_{V}$, and $\omega_{T} \subset \bigcup_{V \in T} \Omega_{V}$. Note that we write $\omega_{i}$ as an abbreviation for $\omega_{V_{i}}$ to avoid more levels of subscripts. Finally, we define the domains

$$
\widetilde{\Omega}_{V}=\bigcup_{V^{\prime} \in \Omega_{V}} \Omega_{V^{\prime}} \quad \text { and } \quad \widetilde{\Omega}_{T}=\bigcup_{V^{\prime} \in T} \widetilde{\Omega}_{V^{\prime}}
$$

containing the neighbor elements of neighbor elements of a vertex $V$ and an element $T$, respectively.

Nédélec [19, 20] finite elements are the natural choice for the approximation of equation (11). For example, the $k^{\text {th }}$ order element of the first family of Nédélec elements generates the space

$$
\mathcal{N}_{h}^{k}=\left\{v \in V:\left.v\right|_{T}=a_{T}+b_{T} \times x \text { with } a_{T}, b_{T} \in\left[P^{k}(T)\right]^{3}\right\} .
$$

The lowest order element $(k=0)$ of this family is the popular edge element. We assume that the finite element space $V_{h} \subset V$ contains the lowest order Nédélec 
space $\mathcal{N}_{h}^{0}$. The finite element approximation to (11) is to find $u_{h} \in V_{h}$ such that

$$
A\left(u_{h}, v_{h}\right)=f\left(v_{h}\right) \quad \forall v_{h} \in V_{h} .
$$

The goal is to derive computable a posteriori error estimators $\eta\left(u_{h}, j\right)$ for the error $\left\|u-u_{h}\right\|_{V}$. In [4], a residual error estimator was derived. As usual, it contains element residuals and jump terms on faces:

$$
\begin{aligned}
\eta_{T}^{2}\left(u_{h}, j\right):= & \frac{h_{T}^{2}}{\nu}\left\|\operatorname{curl} \nu \operatorname{curl} u_{h}+\kappa u_{h}-j\right\|_{L_{2}(T)}^{2}+\frac{h_{T}^{2}}{\kappa}\left\|\operatorname{div} \kappa u_{h}\right\|_{L_{2}(T)}^{2} \\
& +\sum_{F \subset T}\left\{\frac{h_{F}}{\nu}\left\|\left[\nu \operatorname{curl} u_{h}\right]_{t}\right\|_{L_{2}(F)}^{2}+\frac{h_{F}}{\kappa}\left\|\left[\kappa u_{h}\right]_{n}\right\|_{L_{2}(F)}^{2}\right\} .
\end{aligned}
$$

In [4], the efficiency estimate of the error estimator was proven:

$$
\left\|u-u_{h}\right\|_{V}+\text { h.o.t. }(j) \succeq \eta\left(u_{h}, j\right) \text {. }
$$

The reliability estimate

$$
\left\|u-u_{h}\right\|_{V} \preceq \eta\left(u_{h}, j\right)
$$

was proven under the assumption of an $H^{1}$-regular Helmholtz decomposition. This assumption is satisfied for convex or smooth domains, but does not hold true for general Lipschitz domains. The main result of this paper is to prove the reliability estimate for problems on Lipschitz domains. In [25], a Clément-type quasiinterpolation operator was introduced, and a priori estimates were proven. Now, we prove a new approximation error estimate needed for the a posteriori error analysis:

Theorem 1. There exists an operator $\Pi_{h}: H_{D}$ (curl) $\rightarrow \mathcal{N}_{h}^{0}$ with the following properties: For every $u \in H_{D}$ (curl) there exists $\varphi \in H_{D}^{1}$ and $z \in\left[H_{D}^{1}\right]^{3}$ such that

$$
u-\Pi_{h} u=\nabla \varphi+z .
$$

The decomposition satisfies

$$
\begin{aligned}
h_{T}^{-1}\|\varphi\|_{L_{2}(T)}+\|\nabla \varphi\|_{L_{2}(T)} & \leq c\|u\|_{L_{2}\left(\widetilde{\Omega}_{T}\right)}, \\
h_{T}^{-1}\|z\|_{L_{2}(T)}+\|\nabla z\|_{L_{2}(T)} & \leq c\|\operatorname{curl} u\|_{L_{2}\left(\widetilde{\Omega}_{T}\right)} .
\end{aligned}
$$

The constant $c$ depends only on the shape of the elements in the enlarged element patch $\widetilde{\Omega}_{T}$, but does not depend on the global shape of the domain $\Omega$ or the size of the patch $\widetilde{\Omega}_{T}$.

The proof of the theorem is postponed to Section 4 . We note that $\nabla z$ is the $\operatorname{matrix}\left(\frac{\partial z_{i}}{\partial x_{j}}\right)_{i, j=1, \ldots, n}$.

Corollary 2. The residual error estimator is reliable.

Proof. The proof is standard for residual error estimators. The inf - sup stability of $A(.,$.$) and Galerkin orthogonality implies$

$$
\left\|u-u_{h}\right\|_{V} \preceq \sup _{v \in V} \frac{A\left(u-u_{h}, v\right)}{\|v\|_{V}}=\sup _{v \in V} \frac{f\left(v-\Pi_{h} v\right)-A\left(u_{h}, v-\Pi_{h} v\right)}{\|v\|_{V}} .
$$


We apply Theorem 1 to decompose $v-\Pi_{h} v=\nabla \varphi+z$ satisfying the corresponding norm estimates, and bound

$$
\begin{aligned}
f(v- & \left.\Pi_{h} v\right)-A\left(u_{h}, v-\Pi_{h} v\right) \\
= & \int_{\Omega} j(\nabla \varphi+z)-\int_{\Omega} \nu \operatorname{curl} u_{h} \operatorname{curl} z-\int_{\Omega} \kappa u_{h}(\nabla \varphi+z) d x \\
= & \sum_{T \in \mathcal{T}} \int_{T}\left(j-\operatorname{curl} \nu \operatorname{curl} u_{h}-\kappa u_{h}\right) z d x+\sum_{T \in \mathcal{T}} \int_{T} \operatorname{div} \kappa u_{h} \varphi d x \\
& +\sum_{F \in \mathcal{F}} \int_{F}\left[\nu \operatorname{curl} u_{h}\right]_{t} z_{t} d s+\sum_{F \in \mathcal{F}} \int_{F}\left[\kappa u_{h}\right]_{n} \varphi d s \\
\leq & \sum_{T \in \mathcal{T}} \frac{h_{T}}{\sqrt{\nu}}\left\|j-\operatorname{curl} \nu \operatorname{curl} u_{h}-\kappa u_{h}\right\|_{L_{2}(T)} \frac{\sqrt{\nu}}{h_{T}}\|z\|_{L_{2}(T)} \\
& +\sum_{T \in \mathcal{T}} \frac{h_{T}}{\sqrt{\kappa}}\left\|\operatorname{div} \kappa u_{h}\right\|_{L_{2}(T)} \frac{\sqrt{\kappa}}{h_{T}}\|\varphi\|_{L_{2}(T)} \\
& +\sum_{F \in \mathcal{F}} \sqrt{\frac{h_{F}}{\nu}}\left\|\left[\nu \operatorname{curl} u_{h}\right]_{t}\right\|_{L_{2}(F)} \sqrt{\frac{\nu}{h_{F}}}\|z\|_{L_{2}(F)} \\
& +\sum_{F \in \mathcal{F}} \sqrt{\frac{h_{F}}{\kappa}}\left\|\left[\kappa u_{h}\right]_{n}\right\|_{L_{2}(F)} \sqrt{\frac{\kappa}{h_{F}}}\|\varphi\|_{L_{2}(F)} \\
\leq & \eta\left(u_{h}, j\right)\left(\nu\|\operatorname{curl} v\|_{L_{2}}^{2}+\kappa\|v\|_{L_{2}}^{2}\right)^{1 / 2} .
\end{aligned}
$$

In the last step, we have used the trace theorem $\frac{1}{h_{F}}\|z\|_{L_{2}(F)}^{2} \preceq \frac{1}{h_{F}^{2}}\|z\|_{L_{2}(T)}^{2}+$ $\|\nabla z\|_{L_{2}(T)}^{2}$, where $T$ is an element containing the face $F$.

\section{Commuting QuASI-INTERPolation OPERATORS}

To study interpolation operators in $H$ (curl) it is an advantage to consider the whole sequence of spaces $H^{1}, H$ (curl), $H$ (div) and $L_{2}$. The corresponding lowest order finite elements are continuous and piecewise linear elements $\mathcal{L}_{h}^{1}$ with the vertex basis $\left\{\varphi_{V}\right\}$ for $H^{1}$, the Nédélec elements $\mathcal{N}_{h}^{0}$ with the edge basis $\left\{\varphi_{E}\right\}$ for $H$ (curl), the Raviart Thomas elements $\mathcal{R} T_{h}^{0}$ with the face basis $\left\{\varphi_{F}\right\}$ in $H$ (div), and piece-wise constant elements $\mathcal{S}_{h}^{0}$ with the element basis $\left\{\varphi_{T}\right\}$ for $L_{2}$. The basis functions are chosen biorthogonal to the canonical degrees of freedom, i.e., $\varphi_{V_{j}}\left(V_{i}\right)=\delta_{i, j}, \int_{E_{i}} \varphi_{E_{j}} \cdot t_{i} d s=\delta_{i, j}, \int_{F_{i}} \varphi_{F_{j}} \cdot n_{i} d s=\delta_{i, j}$, and $\int_{T_{i}} \varphi_{T_{j}} d x=\delta_{i, j}$.

In [25, quasi-interpolation operators for all these spaces were constructed which satisfy the commuting diagram properties

$$
\nabla \Pi_{h}^{V}=\Pi_{h}^{E} \nabla, \quad \operatorname{curl} \Pi_{h}^{E}=\Pi_{h}^{F} \text { curl, } \quad \operatorname{div} \Pi_{h}^{F}=\Pi_{h}^{T} \operatorname{div},
$$

which are visualized in the de Rham complex as

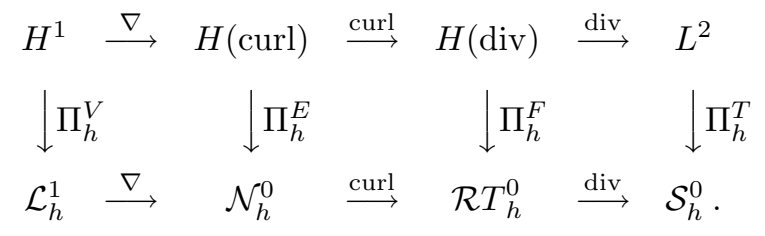


For smooth functions, classical nodal interpolation operators can be applied. These are defined as

$$
\begin{aligned}
\left(I_{h}^{V} w\right)(x) & :=\sum_{V \in \mathcal{V}} w(V) \varphi_{V}(x), \\
\left(I_{h}^{E} v\right)(x) & :=\sum_{E \in \mathcal{E}} \int_{E} v \cdot t_{E} d s \varphi_{E}(x), \\
\left(I_{h}^{F} q\right)(x) & :=\sum_{F \in \mathcal{F}} \int_{F} q \cdot n_{F} d s \varphi_{F}(x), \\
\left(I_{h}^{T} s\right)(x) & :=\sum_{T \in \mathcal{T}} \int_{T} s d x \varphi_{T}(x) .
\end{aligned}
$$

A quasi-interpolation operator for $H^{1}$ functions is defined by local averaging. For each vertex $V$, fix a function $f_{V} \in L_{2}\left(\omega_{V}\right)$ such that $\int_{\omega_{V}} f_{V}(y) d y=1$ and $\left\|f_{V}\right\|_{L_{2}} \simeq h^{-3 / 2}$. One possible choice is $f=\frac{1}{\left|\omega_{V}\right|}$. Then, the quasi-interpolation operator is defined as

$$
\Pi_{h}^{V} w=\sum_{V}\left(\int_{\omega_{V}} f_{V}(y) w(y) d y\right) \varphi_{V}
$$

The quasi-interpolation operator is well defined for $w \in L_{2}(\Omega)$. Due to the integral constraint on $f_{V}$, the quasi-interpolation operator preserves constant functions.

To deal with boundary conditions, we propose a modification for the vertices on the boundary. Let $\widetilde{\Omega}$ be an enlarged domain, and let $\Omega_{D}$ be an outer neighborhood of the essential boundary $\Gamma_{D}$; see Figure凹 in Appendix A. The function $w \in H_{D}^{1}(\Omega)$ is continuously extended to $\widetilde{w} \in \widetilde{\Omega}$. The extension is such that $\widetilde{w}=0$ in $\Omega_{D}$. In Appendix A we introduce such extension procedures for all involved function spaces.

If $V$ is a vertex on the essential boundary $\Gamma_{D}$, we choose $\omega_{V} \subset \Omega_{D}$, again with $\left|\omega_{V}\right| \simeq h_{V}^{3}$. Thus, the interpolation function preserves zero boundary values. If $V$ is on the natural boundary, we may choose $\omega_{V} \subset \widetilde{\Omega}$ such that $\left.\widetilde{w}\right|_{\omega_{V}}$ depends on $\left.w\right|_{\Omega_{V}}$ only.

This class of averaging operators was extended to the other function spaces in [25]. Now, we give a different definition for the same operators. We define the quasi-interpolation operator as the composition of the classical interpolation operator, and a smoothing operator $S$

$$
\Pi_{h}=I_{h} S .
$$

Let the point $x$ be contained in the tetrahedral element $T=\left[V_{T_{1}}, V_{T_{2}}, V_{T_{3}}, V_{T_{4}}\right]$. By means of its barycentric coordinates $\lambda_{1}(x), \ldots, \lambda_{4}(x)$, it is represented as

$$
x=\sum_{j=1}^{4} \lambda_{j}(x) V_{T_{j}} .
$$

Now, let $y_{j} \in \omega_{T_{j}}$. Define $\hat{x}$ by the same barycentric coordinates with respect to the tetrahedron $\left[y_{1}, \ldots, y_{4}\right]$ :

$$
\hat{x}\left(x, y_{1}, y_{2}, y_{3}, y_{4}\right)=\sum_{j=1}^{4} \lambda_{j}(x) y_{j} ;
$$

see Figure 3 , 


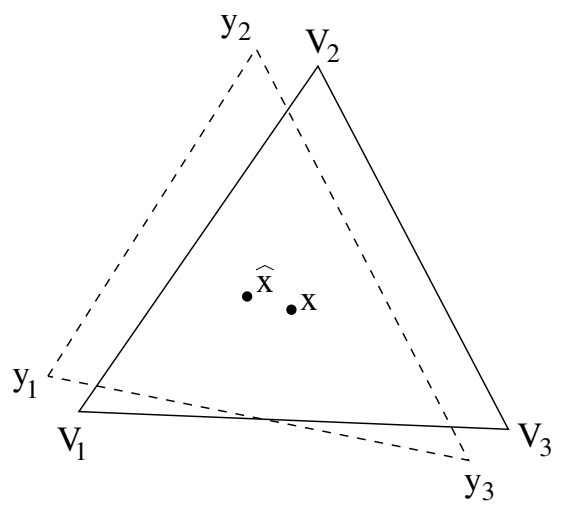

Figure 3. Moved point $\hat{x}$

We define the smoothing operator $S^{V}$ for $H^{1}$ functions as

$$
\left(S^{V} w\right)(x):=\iint_{\omega_{T_{1}}} \int_{\omega_{T_{2}}} \int_{\omega_{T_{3}}} \int_{\omega_{T_{4}}} f_{T_{1}}\left(y_{1}\right) f_{T_{2}}\left(y_{2}\right) f_{T_{3}}\left(y_{3}\right) f_{T_{4}}\left(y_{4}\right) w(\hat{x}) d y_{4} d y_{3} d y_{2} d y_{1} .
$$

If $x$ coincides with a vertex of the element, say, $x=V_{T_{1}}$, then $\lambda_{1}=1$ and $\lambda_{2}=$ $\lambda_{3}=\lambda_{4}=0$, and thus, $\hat{x}=y_{1}$. In this case, the smoothing operator simplifies to

$$
\begin{aligned}
\left(S^{V} w\right)\left(V_{T_{1}}\right) & =\int_{\omega_{T_{1}}} f_{T_{1}}\left(y_{1}\right) w\left(y_{1}\right) d y_{1} \int_{\omega_{T_{2}}} f_{T_{2}}\left(y_{2}\right) d y_{2} \int_{\omega_{T_{3}}} f_{T_{3}}\left(y_{3}\right) d y_{3} \int_{\omega_{T_{4}}} f_{T_{4}}\left(y_{4}\right) d y_{4} \\
& =\int_{\omega_{T_{1}}} f_{T_{1}}\left(y_{1}\right) w\left(y_{1}\right) d y_{1} .
\end{aligned}
$$

The nodal interpolation operator $I_{h}^{V}$ requires these vertex values only. Thus, the quasi-interpolation operator $\Pi_{h}^{V}=I_{h}^{V} S^{V}$ is

$$
\Pi_{h}^{V} w=\sum_{V \in \mathcal{V}}\left(S^{V} w\right)\left(V_{i}\right) \varphi_{V}=\sum_{V \in \mathcal{V}_{\omega_{V}}} \int_{f_{V}}(y) w(y) d y \varphi_{V} .
$$

Similarly, if $x$ is on an edge, only the two barycentric coordinates of the vertices on the edge are non-zero, and the quadruple integral simplifies to a double integral. For faces, the integral simplifies to a triple integral involving the vertices of the face. This property ensures continuity of $S^{V} w$ between neighboring elements.

The smoothing operators for the $H$ (curl) is defined by the co-variant transformation

$$
\left(S^{E} u\right)(x):=\int_{\omega_{T_{1}}} \int_{\omega_{T_{2}}} \int_{\omega_{T_{3}}} \int_{\omega_{T_{4}}} f_{T_{1}} f_{T_{2}} f_{T_{3}} f_{T_{4}}\left(\frac{d \hat{x}}{d x}\right)^{T} u(\hat{x}) d y_{4} d y_{3} d y_{2} d y_{1},
$$

the smoothing for $H$ (div) involves the Piola-transformation

$$
\left(S^{F} q\right)(x):=\iint_{\omega_{T_{1}}} \int_{\omega_{T_{2}}} \int_{\omega_{T_{3}}} \int_{\omega_{T_{4}}} f_{T_{1}} f_{T_{2}} f_{T_{3}} f_{T_{4}} \operatorname{det}\left(\frac{d \hat{x}}{d x}\right)\left(\frac{d \hat{x}}{d x}\right)^{-T} q(\hat{x}) d y_{4} d y_{3} d y_{2} d y_{1},
$$


and for the $L_{2}$-case it becomes

$$
\left(S^{T} s\right)(x):=\int_{\omega_{T_{1}}} \int_{\omega_{T_{2}}} \int_{\omega_{T_{3}}} \int_{\omega_{T_{4}}} f_{T_{1}} f_{T_{2}} f_{T_{3}} f_{T_{4}} \operatorname{det}\left(\frac{d \hat{x}}{d x}\right) s(\hat{x}) d y_{4} d y_{3} d y_{2} d y_{1} .
$$

The $H$ (curl) quasi-interpolation operator is

$$
\begin{aligned}
\Pi_{h}^{E} u & =I_{h}^{E} S^{E} u=\sum_{E \in \mathcal{E}} \int_{E}\left(S^{E} u\right)_{t} d s \varphi_{E} \\
& =\sum_{E \in \mathcal{E}_{\omega_{E_{1}}}} \int_{\omega_{E_{2}}} f_{E_{1}} f_{E_{2}} \int_{V_{1}}^{V_{2}}\left[\left(\frac{d \hat{x}}{d x}\right)^{T} u(\hat{x})\right]_{t} d s d y_{1} d y_{2} \varphi_{E} \\
& =\sum_{E \in \mathcal{E}_{\omega_{E_{1}}}} \int_{\omega_{E_{2}}} \int_{E_{1}} f_{E_{2}} \int_{y_{1}}^{y_{2}} u_{t} d s d y_{1} d y_{2} \varphi_{E} .
\end{aligned}
$$

Instead of taking the line integral of the tangential component from $V_{E_{1}}$ to $V_{E_{2}}$, one integrates over all lines from $\omega_{E_{1}}$ to $\omega_{E_{2}}$, and averages. This was the definition in [25. Similarly, the $H($ div) quasi-interpolation operator is a triple-integral over the normal flux over moved faces:

$$
\Pi_{h}^{F} q=\sum_{F \in \mathcal{F}_{\omega_{F_{1}}}} \int_{\omega_{F_{2}}} \int_{\omega_{F_{3}}} \int_{F_{1}} f_{F_{2}} f_{F_{3}} \int_{\left[y_{1}, y_{2}, y_{3}\right]} q_{n} d s d y_{1} d y_{2} d y_{3} \varphi_{F} .
$$

Lemma 3. The smoothing operators commute in the sense of

$$
\begin{aligned}
\nabla S^{V} & =S^{E} \nabla, \\
\operatorname{curl} S^{E} & =S^{F} \text { curl, } \\
\operatorname{div} S^{F} & =S^{T} \operatorname{div} .
\end{aligned}
$$

Proof. We prove the first relation. The other ones use the proper transformation rules for the co-variant and the Piola-transformation:

$$
\begin{aligned}
\left(\nabla S^{V} w\right)(x) & =\iiint \int f_{T_{1}} \ldots f_{T_{4}} \nabla(w(\hat{x})) d y_{4} d y_{3} d y_{2} d y_{1} \\
& \left.=\iiint \int f_{T_{1}} \ldots f_{T_{4}}\left(\frac{d \hat{x}}{d x}\right)^{T}(\nabla w)(\hat{x})\right) d y_{4} d y_{3} d y_{2} d y_{1} \\
& =\left(S^{E} \nabla w\right)(x) .
\end{aligned}
$$

Corollary 4. The quasi-interpolation operators commute in the sense of

$$
\begin{aligned}
\nabla \Pi_{h}^{V} & =\Pi_{h}^{E} \nabla, \\
\operatorname{curl} \Pi_{h}^{E} & =\Pi_{h}^{F} \text { curl, } \\
\operatorname{div} \Pi_{h}^{F} & =\Pi_{h}^{T} \operatorname{div} .
\end{aligned}
$$

Proof. The nodal interpolation operators commute, so the composition $\Pi_{h}=I_{h} S$ also commutes.

Remark 5 . There are several possibilities to choose the weighting functions $f_{V}$ such that the $H^{1}$ operator preserves finite element functions. But, the operators for the other spaces will in general not inherit this projection property. For the purpose of a posteriori error analysis, the projection property is not required. In [27, the operators are modified to obtain projections. 


\section{INTERPOLATION ERROR ESTIMATES FOR THE $\Pi^{E}$}

Before proving Theorem [1, we first analyze the decomposition of the interpolation error into local $H$ (curl) functions.

Theorem 6. There exists a decomposition of the interpolation error

$$
u-\Pi_{h}^{E} u=\sum_{V \in \mathcal{V}} u_{V} \quad \text { with } \quad u_{V} \in H_{D}\left(\operatorname{curl}, \Omega_{V}\right),
$$

where $H_{D}\left(\operatorname{curl}, \Omega_{V}\right)=\left\{v \in H_{D}(\operatorname{curl}): v=0\right.$ in $\left.\Omega \backslash \Omega_{V}\right\}$. This decomposition satisfies the local estimates

$$
\begin{aligned}
\left\|u_{V}\right\|_{L_{2}\left(\Omega_{V}\right)} & \preceq\|u\|_{L_{2}\left(\widetilde{\Omega}_{V}\right)}, \\
\left\|\operatorname{curl} u_{V}\right\|_{L_{2}\left(\Omega_{V}\right)} & \preceq\|\operatorname{curl} u\|_{L_{2}\left(\widetilde{\Omega}_{V}\right)}
\end{aligned}
$$

Proof. We decompose the interpolation error as

$$
u-\Pi_{h}^{E} u=\left(u-S^{E} u\right)+\left(S^{E} u-I_{h}^{E} S^{E} u\right)
$$

and bound the two terms on the right hand side in Lemma 7 and Lemma 10 below.

Lemma 7. There exists a decomposition

$$
u-S^{E} u=\sum_{V \in \mathcal{V}} u_{V} \quad \text { with } \quad u_{V} \in H_{D}\left(\operatorname{curl}, \Omega_{V}\right)
$$

which satisfies the continuity estimates

$$
\begin{aligned}
\left\|u_{V}\right\|_{L_{2}\left(\Omega_{V}\right)} & \preceq\|u\|_{L_{2}\left(\widetilde{\Omega}_{V}\right)}, \\
\left\|\operatorname{curl} u_{V}\right\|_{L_{2}\left(\Omega_{V}\right)} & \preceq\|\operatorname{curl} u\|_{L_{2}\left(\widetilde{\Omega}_{V}\right)} .
\end{aligned}
$$

Proof. We formally extend the quadruple integral of the smoothing operators to an $N$-dimensional integral, where $N$ is the global number of vertices:

$$
S^{V} w(x)=\int_{\omega_{1}} \cdots \int_{\omega_{N}} f_{1}\left(y_{1}\right) \cdots f_{N}\left(y_{n}\right) w(\hat{x}) d y_{N} \cdots d y_{1}
$$

Formally, we write $\hat{x}=\hat{x}\left(x, y_{1}, \ldots y_{N}\right)$. Indeed, $\hat{x}$ depends only on the four (three, two, one) $y_{i}$ corresponding to the vertices of the element (face, edge, vertex, respectively) containing the point $x$. The other integrals $\int_{\omega_{k}} f_{k}\left(y_{k}\right) d y_{k}$ are just constant factors 1 . This extended notation allows the definition of partial smoothing operators

$$
S_{i}^{V} w=\int_{\omega_{1}} \cdots \int_{\omega_{i}} f_{1}\left(y_{1}\right) \cdots f_{i}\left(y_{i}\right) w\left(\hat{x}\left(y_{1}, \ldots y_{i}, V_{i+1}, V_{N}\right)\right) d y_{i} \cdots d y_{1}
$$

We can apply telescoping

$$
w-S^{V} w=\sum_{i=1}^{N}\left(S_{i-1}^{V} w-S_{i}^{V} w\right)
$$

These terms are indeed a local decomposition of $w$. Let $w_{i}:=S_{i-1}^{V} w-S_{i}^{V} w$. If $x$ does not belong to the interior of $\Omega_{V_{i}}$, then $\hat{x}$ does not depend on $y_{i}$, which implies that $w_{i}(x)=0$. In the same way, we define partial smoothing operators for the 
other spaces. Again, the partial smoothing operators commute. It remains to show the $L_{2}$-bounds for the decomposition, namely

$$
\left\|S_{i-1} u-S_{i} u\right\|_{L_{2}\left(\Omega_{V}\right)} \preceq\|u\|_{L_{2}\left(\widetilde{\Omega}_{V}\right)} .
$$

The commutativity immediately implies such bounds for the semi-norms, e.g.,

$$
\left\|\operatorname{curl}\left(S_{i-1}^{E} u-S_{i}^{E} u\right)\right\|_{L_{2}\left(\Omega_{V}\right)}=\left\|\left(S_{i-1}^{F}-S_{i}^{F}\right) \operatorname{curl} u\right\|_{L_{2}\left(\Omega_{V}\right)} \preceq\|\operatorname{curl} u\|_{L_{2}\left(\widetilde{\Omega}_{V}\right)} .
$$

The $L_{2}$ continuity is proven element-wise for $S_{i}$. We show that

$$
\left\|S_{i}^{V} w\right\|_{L_{2}(T)} \preceq\|w\|_{L_{2}\left(\omega_{T}\right)} .
$$

The operator $S_{i}^{V}$ performs smoothing for the vertices $T_{j}$ of the element with $T_{j} \leq i$, but keeps vertices $T_{j}$ with $j>i$ constant. To keep the complexity of the notation reasonable, we assume (w.l.o.g.) that smoothing is performed for the first two vertices, i.e., $T_{1} \leq i, T_{2} \leq i, T_{3}>i$, and $T_{4}>i$. Then, smoothing gives on the element $T$

$$
\left(S_{i}^{V} w\right)(x)=\int_{\omega_{T_{1}}} \int_{\omega_{T_{2}}} f_{T_{1}}\left(y_{1}\right) f_{T_{2}}\left(y_{2}\right) w\left(\hat{x}\left(x, y_{1}, y_{2}, V_{T_{3}}, V_{T_{4}}\right)\right) d y_{2} d y_{1}
$$

We apply the Hölder inequality for $L_{1}-L_{\infty}$ to bound

$$
\begin{array}{rl}
\| S_{i}^{V} & w \|_{L_{2}(T)}^{2} \\
= & \int_{T}\left(\int_{\omega_{T_{1}}} \int_{\omega_{T_{2}}} f_{T_{1}}\left(y_{1}\right) f_{T_{2}}\left(y_{2}\right) w\left(\hat{x}\left(x, y_{1}, y_{2}, V_{T_{3}}, V_{T_{4}}\right)\right) d y_{2} d y_{1}\right)^{2} d x \\
& \leq \int_{T}\left(\int_{\omega_{T_{1}}} \int_{\omega_{T_{2}}}\left|f_{T_{1}}\left(y_{1}\right)\right|\left|f_{T_{2}}\left(y_{2}\right)\right| d y_{2} d y_{1}\right)^{2} \\
& \sup _{\substack{y_{1} \in \omega_{T_{1}} \\
y_{2} \in \omega_{T_{2}}}}\left|w\left(\hat{x}\left(x, y_{1}, y_{2}, V_{T_{3}}, V_{T_{4}}\right)\right)\right|^{2} d x \\
= & \left\|f_{T_{1}}\right\|_{L_{1}\left(\omega_{T_{1}}\right)}^{2}\left\|f_{T_{2}}\right\|_{L_{1}\left(\omega_{T_{2}}\right)}^{2} \sup _{\substack{y_{1} \in \omega_{T_{1}} \\
y_{2} \in \omega_{T_{2}}}} \int_{T} w\left(\hat{x}\left(x, y_{1}, y_{2}, V_{T_{3}}, V_{T_{4}}\right)\right)^{2} d x .
\end{array}
$$

There holds $\left\|f_{T_{1}}\right\|_{L_{1}\left(\omega_{T_{1}}\right)} \leq\left\|f_{T_{1}}\right\|_{L_{2}\left(\omega_{T_{1}}\right)}\left|\omega_{T_{1}}\right|^{1 / 2} \preceq 1$. The integral in the last term is transformed to the moved tetrahedron $\hat{x}\left(T, y_{1}, y_{2}, V_{T_{3}}, V_{T_{4}}\right)$ :

$$
\begin{aligned}
& \int_{T} w\left(\hat{x}\left(x, y_{1}, y_{2}, V_{T_{3}}, V_{T_{4}}\right)\right)^{2} d x \\
& \quad=\int_{\hat{x}\left(T, y_{1}, y_{2}, V_{T_{3}}, V_{T_{4}}\right)} w(\xi)^{2} \operatorname{det}\left(\frac{d \hat{x}}{d x}\right)^{-1} d \xi \\
& \quad \preceq\|w\|_{L_{2}\left(\hat{x}\left(T, y_{1}, y_{2}, V_{T_{3}}, V_{T_{4}}\right)\right)}^{2} \leq\|w\|_{L_{2}(\omega T)}^{2} .
\end{aligned}
$$

We have used the fact that $\frac{d \hat{x}}{d x}$ as well as its inverse is bounded by a constant due to the sufficiently separated domains $\omega_{V}$. The $L_{2}$-estimates for the other smoothing operators are proven in the same way.

We have already observed that the smoothing operator $S^{V}$ provides well defined vertex values. Similarly, the other smoothing operators also provide well defined values at some of the lower dimensional objects. 
Lemma 8. The smoothed functions have well defined boundary values in the following sense:

$$
\begin{aligned}
\left\|S^{V} w\right\|_{L_{2}(V)}^{2} & \preceq h^{-3}\|w\|_{L_{2}\left(\omega_{V}\right)}^{2}, \\
\left\|S^{V} w\right\|_{L_{2}(E)}^{2} & \preceq h^{-2}\|w\|_{L_{2}\left(\omega_{E}\right)}^{2}, \\
\left\|S^{V} w\right\|_{L_{2}(F)}^{2} & \preceq h^{-1}\|w\|_{L_{2}\left(\omega_{F}\right)}^{2}, \\
\left\|\left(S^{E} u\right)_{t}\right\|_{L_{2}(E)}^{2} & \preceq h^{-2}\|u\|_{L_{2}\left(\omega_{E}\right)}^{2}, \\
\left\|\left(S^{E} u\right)_{t}\right\|_{L_{2}(F)}^{2} & \preceq h^{-1}\|u\|_{L_{2}\left(\omega_{F}\right)}^{2}, \\
\left\|\left(S^{F} q\right)_{n}\right\|_{L_{2}(F)}^{2} & \preceq h^{-1}\|q\|_{L_{2}\left(\omega_{F}\right)}^{2} .
\end{aligned}
$$

Proof. We prove $\left\|S^{V} w\right\|_{L_{2}(F)}^{2} \preceq h^{-1}\|w\|_{L_{2}\left(\omega_{F}\right)}^{2}$. The other estimates follow with the same arguments. The face $F$ is split into three parts, $F_{\lambda_{1}}, F_{\lambda_{2}}, F_{\lambda_{3}}$, according to

$$
F_{\lambda_{i}}=\left\{x: \lambda_{i}(x)=\max \left\{\lambda_{1}(x), \lambda_{2}(x), \lambda_{3}(x)\right\}\right\} .
$$

We apply Cauchy-Schwarz on $\omega_{F_{1}}$, and the $L_{1}-L_{\infty}$ Hölder inequality on $\omega_{F_{2}}$ and $\omega_{F_{3}}$ to bound

$$
\begin{aligned}
& \left\|S^{V} w\right\|_{L_{2}\left(F_{\lambda_{1}}\right)}^{2} \\
& =\int_{F_{\lambda_{1}}}\left(\int_{\omega_{F_{1}}} \int_{\omega_{F_{2}}} \int_{\omega_{F_{3}}} f_{1}\left(y_{1}\right) f_{2}\left(y_{2}\right) f_{3}\left(y_{3}\right) w\left(\hat{x}\left(x, y_{1}, y_{2}, y_{3}\right)\right) d y_{3} d y_{2} d y_{1}\right)^{2} d x \\
& \leq\left\|f_{1}\right\|_{L_{2}}^{2}\left\|f_{2}\right\|_{L_{1}}^{2}\left\|f_{3}\right\|_{L_{1}}^{2} \sup _{y_{2}, y_{3}} \int_{F_{\lambda_{1}}} \int_{\omega_{F_{1}}}\left|w\left(\hat{x}\left(x, y_{1}, y_{2}, y_{3}\right)\right)\right|^{2} d y_{1} d x \\
& \preceq h_{T}^{-3} \sup _{y_{2}, y_{3}} \int_{F_{\lambda_{1}}} \int_{\hat{x}\left(x, \omega_{F_{1}}, y_{2}, y_{3}\right)} w(\eta)^{2} \operatorname{det}\left(\frac{d \hat{x}}{d y_{1}}\right)^{-1} d \eta d x .
\end{aligned}
$$

The transformation is $\hat{x}\left(x, y_{1}, y_{2}, y_{3}\right)=\sum_{i=1}^{3} \lambda_{i}(x) y_{i}$. Thus, $\frac{d \hat{x}}{d y_{1}}=\lambda_{1}(x) I$. On $F_{\lambda_{1}}$ there is $\lambda_{1} \in\left[\frac{1}{3}, 1\right]$, and thus $\operatorname{det} \frac{d \hat{x}}{d y_{1}} \simeq 1$. Insert this to obtain

$$
\left\|S^{V} w\right\|_{L_{2}\left(F_{\lambda_{1}}\right)}^{2} \preceq h_{T}^{-3} \int_{F_{\lambda_{1}}} \int_{\omega_{F}} w(\eta)^{2} d \eta d x \preceq h_{T}^{-1}\|w\|_{L_{2}\left(\omega_{F}\right)}^{2} .
$$

The $L_{2}$-norm on the other two parts, $F_{\lambda_{2}}$ and $F_{\lambda_{3}}$, follow from permutation.

Lemma 9. There exists an extension operator

$$
E^{E}: H_{0}^{1}(E) \rightarrow H_{0}^{1}\left(\Omega_{E}\right)
$$

which is continuous in the sense of

$$
\begin{aligned}
\left\|E^{E} w\right\|_{H^{1}\left(\Omega_{E}\right)}+h^{1 / 2}\left\|E^{E} w\right\|_{H^{1}(F)} & \preceq h\|w\|_{H^{1}(E)}, \\
\left\|E^{E} w\right\|_{L_{2}\left(\Omega_{E}\right)}+h^{1 / 2}\left\|E^{E} w\right\|_{L_{2}(F)} & \preceq h\|w\|_{L_{2}(E)} .
\end{aligned}
$$

Here, $F$ is an arbitrary face inside $\Omega_{E}$. There exists an extension operator

$$
E^{F}: H_{0}^{1}(F) \rightarrow H_{0}^{1}\left(\Omega_{F}\right)
$$

which is continuous in the sense of

$$
\begin{aligned}
\left\|E^{F} w\right\|_{H^{1}\left(\Omega_{F}\right)} & \leq h^{1 / 2}\|w\|_{H^{1}(F)}, \\
\left\|E^{F} w\right\|_{L_{2}\left(\Omega_{F}\right)} & \leq h^{1 / 2}\|w\|_{L_{2}(F)} .
\end{aligned}
$$


Proof. Let $w \in H_{0}^{1}(E)$. We construct the extension onto an element $T$ sharing the edge $E$. Let $\lambda_{E_{1}}$ and $\lambda_{E_{2}}$ be the two barycentric coordinates of the vertices connected by the edge, and set $\lambda_{E}=\lambda_{E_{1}}+\lambda_{E_{2}}$.

The extension $E^{E} w$ is defined by

$$
E^{E} w(x)=\lambda_{E} w(\hat{x}) \quad \text { with } \quad \hat{x}=\sum_{i=1}^{2} \frac{\lambda_{E_{i}}}{\lambda_{E}} V_{E_{i}} .
$$

Product and chain rule lead to

$$
\nabla E^{E} w(x)=\nabla \lambda_{E} w(\hat{x})+\lambda_{E} \nabla_{t} w(\hat{x}) \frac{d \hat{x}}{d x} .
$$

Observe that $\left|\nabla \lambda_{i}\right| \preceq h^{-1}$, and

$$
\lambda_{E} \frac{d \hat{x}}{d x}=\lambda_{E} \frac{d}{d x} \frac{\lambda_{E_{1}}\left(V_{E_{1}}-V_{E_{2}}\right)}{\lambda_{E}}=\left(\nabla \lambda_{E_{1}}-\frac{\lambda_{E_{1}}}{\lambda_{E}} \nabla \lambda_{E}\right)\left(V_{E_{1}}-V_{E_{2}}\right) .
$$

From $\left|V_{E_{1}}-V_{E_{2}}\right| \preceq h$ there follows $\left|\lambda_{E} \frac{d \hat{x}}{d x}\right| \preceq 1$. This leads to

$$
\left|\nabla E^{E} w(x)\right| \preceq h^{-1}|w(\hat{x})|+\left|\nabla_{t} w(\hat{x})\right| .
$$

With the transformation of integrals and a Friedrichs' inequality on the edge we observe that

$$
\left\|\nabla E^{E} w\right\|_{L_{2}(T)}^{2} \preceq h^{-1}\|w(\hat{x}(x))\|_{L_{2}(T)}+\left\|\nabla_{t} w(\hat{x}(x))\right\|_{L_{2}(T)}^{2} \preceq h\left\|\nabla_{t} w\right\|_{L_{2}(E)} .
$$

The $L_{2}$ estimate and the estimates on faces is left to the reader. Similarly, we define the extension operator from faces by

$$
E^{F} w(x)=\lambda_{F} w(\hat{x}) \quad \text { with } \quad \hat{x}=\sum_{i=1}^{3} \frac{\lambda_{F_{i}}}{\lambda_{F}} V_{F_{i}},
$$

where $F_{1}, F_{2}$, and $F_{3}$ are the vertices of the face, and $\lambda_{F}=\sum_{i=1}^{3} \lambda_{F_{i}}$. The continuity estimates follow with the same arguments.

Lemma 10. There exists a decomposition

$$
S^{E} u-I_{h}^{E} S^{E} u=\sum_{V \in \mathcal{V}} u_{V} \quad \text { with } \quad u_{V} \in H_{D}\left(\operatorname{curl}, \Omega_{V}\right)
$$

which satisfies the continuity estimates

$$
\begin{aligned}
\left\|u_{V}\right\|_{L_{2}\left(\Omega_{V}\right)} & \preceq\|u\|_{L_{2}\left(\widetilde{\Omega}_{V}\right)}, \\
\left\|\operatorname{curl} u_{V}\right\|_{L_{2}\left(\Omega_{V}\right)} & \preceq\|\operatorname{curl} u\|_{L_{2}\left(\widetilde{\Omega}_{V}\right)} .
\end{aligned}
$$

Proof. Since $S^{E} u \in L_{2}(E)$, the nodal edge interpolator is well defined. Set $u_{2}:=$ $S^{E} u-I_{h}^{E} S^{E} u$. It satisfies the continuity estimates

$$
\begin{aligned}
h\left\|u_{2, t}\right\|_{L_{2}(E)} & \preceq\|u\|_{\omega_{E}}, \\
h^{1 / 2}\left\|u_{2, t}\right\|_{L_{2}(F)} & \preceq\|u\|_{\omega_{F}}, \\
\left\|u_{2}\right\|_{L_{2}(T)} & \preceq\|u\|_{\omega_{T}} .
\end{aligned}
$$

Integrating the tangential component of $u_{2}$ along the edge $E=\left[E_{1}, E_{2}\right]$ results in

$$
\Phi_{E}(x):=\int_{E_{1}}^{x} u_{2, t} d s .
$$


Due to zero mean, $\Phi_{E} \in H_{0}^{1}(E)$. Using the extension from edges of Lemma 9, we construct

$$
u_{3}=u_{2}-\sum_{E \in \mathcal{E}} \nabla E^{E} \Phi_{E}
$$

Each of the terms $\nabla E^{E} \Phi_{E}$ can be included in one of the terms of the decomposition (10). The remaining $u_{3}$ satisfies

$$
\begin{aligned}
h^{1 / 2}\left\|u_{3, t}\right\|_{L_{2}(F)} & \preceq\|u\|_{\omega_{F}}, \\
\left\|u_{3}\right\|_{L_{2}(T)} & \preceq\|u\|_{\omega_{T}} .
\end{aligned}
$$

By commutativity, the following estimates are also obtained for curl $u$ :

$$
\begin{aligned}
h^{1 / 2}\left\|\left(\operatorname{curl} u_{3}\right)_{n}\right\|_{L_{2}(F)} & \preceq\|\operatorname{curl} u\|_{\omega_{F}}, \\
\left\|\operatorname{curl} u_{3}\right\|_{L_{2}(T)} & \preceq\|\operatorname{curl} u\|_{\omega_{T}} .
\end{aligned}
$$

Next, we extend from faces. For this, decompose $u_{3, t} \in H_{0}(\operatorname{curl}, F)$ into

$$
\left.u_{3, t}\right|_{F}=\left(\nabla \Phi_{F}+z_{F}\right)_{t}
$$

such that $\Phi_{F} \in H_{0}^{1}(F)$ and $z_{F} \in\left[H_{0}^{1}(F)\right]^{3}$ satisfy

$$
\begin{aligned}
\left\|\nabla_{t} \phi_{F}\right\|_{L_{2}}+\left\|z_{F}\right\|_{L_{2}} & \preceq\left\|u_{3, t}\right\|_{L_{2}}, \\
\left\|\nabla_{t} z_{F}\right\|_{L_{2}(F)} & \preceq\left\|\operatorname{curl} u_{3, t}\right\| .
\end{aligned}
$$

This is possible due to the two-dimensional version of 22, Lemma 2.2. Both functions, $\Phi_{F}$ and $z_{F}$, are extended by $E^{F}$ onto the adjacent elements. These terms match the decomposition (10) and satisfy the continuity estimates

$$
\left\|\nabla E^{F} \Phi_{F}+E^{F} z_{F}\right\|_{L_{2}\left(\Omega_{F}\right)} \preceq h^{1 / 2}\left\|\left(S^{E} u\right)_{t}\right\|_{L_{2}(F)} \preceq\|u\|_{\omega_{F}}
$$

and

$$
\left\|\operatorname{curl} E^{F} z_{F}\right\|_{L_{2}\left(\Omega_{F}\right)} \preceq h^{1 / 2}\left\|\operatorname{curl}\left(S^{E} u\right)_{t}\right\|_{L_{2}(F)} \preceq\|\operatorname{curl} u\|_{\omega_{F}} .
$$

Finally, define

$$
u_{4}=u_{3}-\sum_{F \in \mathcal{F}}\left\{\nabla E^{F} \Phi_{F}+E^{F} z_{F}\right\}
$$

which has vanishing tangential trace on all faces, and thus splits into local terms.

By the same techniques, one also proves a decomposition result for the space $H$ (div). It might be useful for the analysis of a posteriori error estimators for mixed methods involving the space $H$ (div) such as in [10.

Theorem 11. There exists a decomposition of the interpolation error

$$
q-\Pi_{h}^{F} q=\sum_{V \in \mathcal{V}} q_{V} \quad \text { with } \quad q_{V} \in H_{D}\left(\operatorname{div}, \Omega_{V}\right),
$$

where $H_{D}\left(\operatorname{div}, \Omega_{V}\right)=\left\{v \in H_{D}(\operatorname{div}): v=0\right.$ in $\left.\Omega \backslash \Omega_{V}\right\}$. This decomposition satisfies the local estimates

$$
\begin{aligned}
\left\|q_{V}\right\|_{L_{2}\left(\Omega_{V}\right)} & \preceq\|q\|_{L_{2}\left(\widetilde{\Omega}_{V}\right)}, \\
\left\|\operatorname{div} q_{V}\right\|_{L_{2}\left(\Omega_{V}\right)} & \preceq\|\operatorname{div} q\|_{L_{2}\left(\widetilde{\Omega}_{V}\right)} .
\end{aligned}
$$

Now, we are ready to prove our main result: 
Proof of Theorem 1. Let $u=\sum u_{V}$ be the decomposition of Theorem [6] First, assume that $V$ is an inner vertex or a vertex on the Dirichlet boundary. Then $u_{V} \in H_{0}\left(\operatorname{curl}, \Omega_{V}\right)$. According to [22, Lemma 2.2, there exists a decomposition

$$
u_{V}=\nabla \varphi_{V}+z_{V}
$$

with $\varphi_{V} \in H_{0}^{1}\left(\Omega_{V}\right)$ and $z_{V} \in\left[H_{0}^{1}\left(\Omega_{V}\right)\right]^{3}$. The decomposition is bounded by

$$
\begin{aligned}
h_{V}^{-1}\left\|\varphi_{V}\right\|_{L_{2}\left(\Omega_{V}\right)}+\left\|\nabla \varphi_{V}\right\|_{L_{2}\left(\Omega_{V}\right)} & \preceq\left\|u_{V}\right\|_{L_{2}\left(\Omega_{V}\right)}, \\
h_{V}^{-1}\left\|z_{V}\right\|_{L_{2}\left(\Omega_{V}\right)}+\left\|\nabla z_{V}\right\|_{L_{2}\left(\Omega_{V}\right)} & \preceq\left\|\operatorname{curl} u_{V}\right\|_{L_{2}\left(\Omega_{V}\right)},
\end{aligned}
$$

where the involved constants depend only on the shape of the local domain $\Omega_{V}$. If the vertex is on the Neumann boundary, then $u_{V, t}$ does not necessarily vanish on the boundary of $\Omega_{V}$ which is also the domain boundary. Since the domain is Lipschitz, the whole patch $\Omega_{V}$ can be mirrored over the domain boundary to obtain $\widetilde{\Omega}_{V}$. The function is extended by the co-variant transformation to $H_{0}\left(\operatorname{curl}, \widetilde{\Omega}_{V}\right)$. Now, the above decomposition can be applied.

We define

$$
\varphi=\sum_{V \in \mathcal{V}} \varphi_{V} \quad \text { and } \quad z=\sum_{V \in \mathcal{V}} z_{V}
$$

to obtain the claimed decomposition (2)

$$
u-\Pi_{h}^{E} u=\nabla \varphi+z .
$$

The norm bounds follow from the finite number of overlapping patches.

\section{Appendix A. Commuting extension operators}

We establish extension operators for the spaces $H$ (curl) and $H$ (div) which are bounded in the $L_{2}$ norm and in the corresponding semi-norms. The extended function vanishes on an outer neighborhood of the Dirichlet boundary. We introduce a continuous bijection $x \mapsto \tilde{x}(x)$ between the inner $\left(\Omega_{i}\right)$ and outer $\left(\Omega_{o}\right)$ neighborhoods of the boundary $\partial \Omega$; see Figure 4 . The transformation shall fulfill

$$
\tilde{x}(x)=x \quad \forall x \in \Gamma_{N}
$$

and is bounded in the sense

$$
\left\|\frac{d \tilde{x}}{d x}\right\|_{L_{\infty}} \leq c \quad \text { and } \quad\left\|\left(\frac{d \tilde{x}}{d x}\right)^{-1}\right\|_{L_{\infty}} \leq c .
$$

On Dirichlet boundaries, we shift the exterior domain $\Omega_{0}$ away from the boundary to obtain the domain $\Omega_{D}$ between $\Gamma_{D}$ and $\tilde{x}\left(\Gamma_{D}\right)$. Let $\widetilde{\Omega}=\bar{\Omega} \cup \bar{\Omega}_{D} \cup \Omega_{o}$.

We sketch this construction for general Lipschitz domains. Let $U_{1}, \ldots, U_{M}$ be an open covering of the boundary $\partial \Omega$. Assume that a strip $S$ of with $s$ along $\partial \Omega$ is contained in $\bigcup U_{i}$. Let $\left(e_{\xi_{i}}, e_{\eta_{i}}, e_{\zeta_{i}}\right)$ be local coordinate systems, and let $\varphi_{i}\left(\xi_{i}, \eta_{i}\right)$ be Lipschitz functions such that $U_{i} \cap \Omega=\left\{\left(\xi_{i}, \eta_{i}, \zeta_{i}\right) \in U_{i}: \zeta_{i}>\varphi_{i}\left(\xi_{i}, \eta_{i}\right)\right\}$. Define the limited distance function to the non-Dirichlet boundary as $d(x):=$ $\min \left\{s / 2, \operatorname{dist}\left\{x, \Gamma_{N}\right\}\right\}$. Now, we can define the mirroring operator with shift for the Dirichlet boundary: Assume $x \in U_{i} \cap \Omega$ has the local coordinates $\left(\xi_{i}, \eta_{i}, \zeta_{i}\right)$. The vertical projection to the boundary $x_{i}^{b}(x)$ is defined by the local coordinates $\left(\xi_{i}, \eta_{i}, \varphi_{i}\left(\xi_{i}, \eta_{i}\right)\right)$, and $\tilde{x}_{i}(x)=x_{b}-\left(\left|x_{i}^{b}-x\right|+d\left(x_{b}\right)\right) e_{\zeta_{i}}$. Finally, introduce a partition of unity $\left\{\psi_{i}\right\}$ such that $\sum \psi_{i}=1$ on $\partial \Omega$, and set $\tilde{x}(x):=\sum \psi_{i}\left(x_{i}^{b}\right) \tilde{x}_{i}(x)$. 


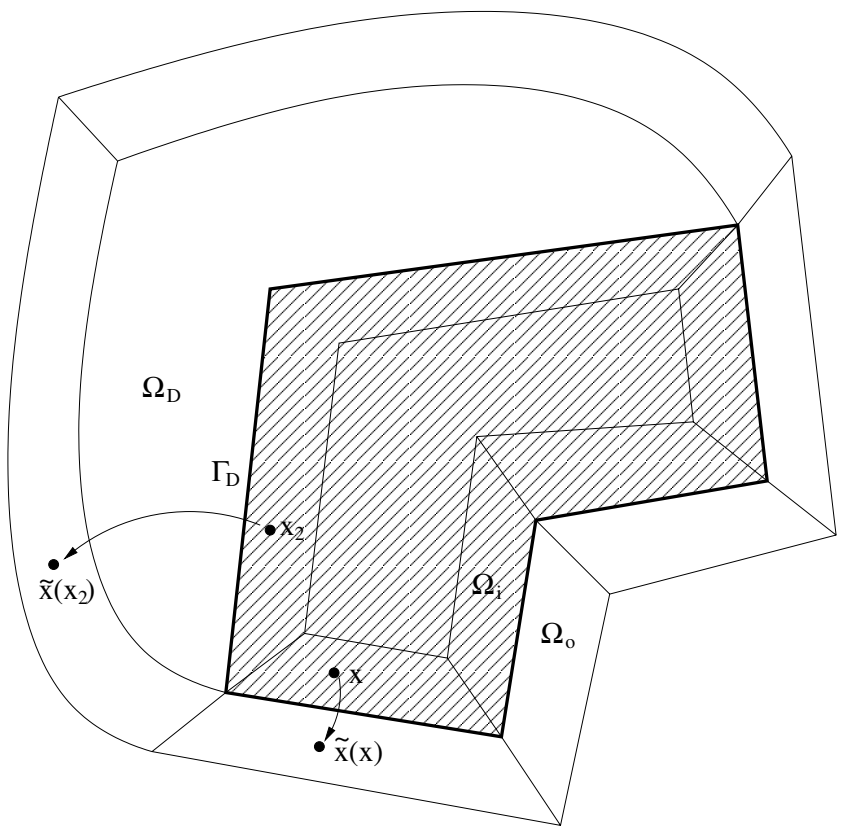

FiguRE 4. Transformation for extension

The extension for $H^{1}$ functions is defined by mirroring:

$$
\tilde{w}(x)=\left\{\begin{array}{cl}
w(x), & x \in \Omega, \\
0, & x \in \Omega_{D}, \\
w\left(\tilde{x}^{-1}(x)\right), & x \in \Omega_{o} .
\end{array}\right.
$$

Using the chain rule, its piece-wise gradient evaluates to

$$
\nabla \tilde{w}(x)=\left\{\begin{array}{cl}
\nabla w(x), & x \in \Omega, \\
0, & x \in \Omega_{D}, \\
\left(\tilde{x}^{\prime}\right)^{-T}(\nabla w)\left(\tilde{x}^{-1}(x)\right), & x \in \Omega_{o} .
\end{array}\right.
$$

Since the extension has continuous traces on the interfaces between $\Omega_{i}, \Omega_{o}$, and $\Omega_{D}$, the piece-wise gradient is also the global gradient of $\tilde{w}$. We have assumed that $\tilde{x}^{\prime}$ as well as its inverse is in $L_{\infty}$. This ensures that the extension is bounded with respect to the $L_{2}$-norm. It also ensures that the gradient of the extension is bounded by the $L_{2}$-norm of the gradient, i.e., the extension is bounded in the $H^{1}$-semi-norm.

Motivated by the commuting diagram, the extension $\tilde{u}$ of an $H$ (curl) function $u$ is defined like the extension of gradients:

$$
\tilde{u}(x)=\left\{\begin{array}{cl}
u(x), & x \in \Omega, \\
0, & x \in \Omega_{D}, \\
\left(\tilde{x}^{\prime}\right)^{-T} u\left(\tilde{x}^{-1}(x)\right), & x \in \Omega_{o} .
\end{array}\right.
$$

With this so-called co-variant transformation for the function $u$, the transformation of its curl evaluates to the Piola-transformation:

$$
\operatorname{curl} \tilde{u}(x)=\left\{\begin{array}{cl}
\operatorname{curl} u(x), & x \in \Omega, \\
0, & x \in \Omega_{D}, \\
\operatorname{det}\left(\tilde{x}^{\prime}\right)^{-1}\left(\tilde{x}^{\prime}\right) \operatorname{curl} u\left(\tilde{x}^{-1}(x)\right), & x \in \Omega_{o} .
\end{array}\right.
$$


The extension $\tilde{u}$ has continuous tangential traces ensuring that $\tilde{u} \in H(\operatorname{curl}, \widetilde{\Omega})$. Since the curl of the extended function depends continuously only on the curl of the original function, the extension is bounded in the curl semi-norm. In the same fashion, we define the extension of $H($ div) functions $q$ by the Piola-transformation:

$$
\tilde{q}(x)=\left\{\begin{array}{cl}
q(x), & x \in \Omega, \\
0, & x \in \Omega_{D}, \\
\operatorname{det}\left(\tilde{x}^{\prime}\right)^{-1}\left(\tilde{x}^{\prime}\right) q\left(\tilde{x}^{-1}(x)\right), & x \in \Omega_{o} .
\end{array}\right.
$$

This one provides continuous normal traces. Now, forming the divergence leads to

$$
\operatorname{div} \tilde{q}(x)=\left\{\begin{array}{cl}
\operatorname{div} q(x), & x \in \Omega, \\
0, & x \in \Omega_{D}, \\
\operatorname{det}\left(\tilde{x}^{\prime}\right)^{-1} \operatorname{div} q\left(\tilde{x}^{-1}(x)\right), & x \in \Omega_{o} .
\end{array}\right.
$$

We also take this one for the extension of $L_{2}$-functions.

\section{REFERENCES}

[1] M. Ainsworth and J. Coyle. Hierarchic finite element bases on unstructured tetrahedral meshes. Int. J. Num. Meth. Eng., 58(14), 2103-2130. MR2022172(2004j:65178)

[2] M. Ainsworth and T. Oden. A Posteriori Error Estimation in Finite Element Analysis. Wiley-Interscience, 2000. MR 1885308 (2003b:65001)

[3] D. N. Arnold, R. S. Falk, and R. Winther. Multigrid in $H$ (div) and H(curl). Numer. Math., 85:197-218, 2000. MR 1754719 (2001d:65161)

[4] R. Beck, R. Hiptmair, R. Hoppe, and B. Wohlmuth. Residual based a posteriori error estimators for eddy current computations. $M^{2} A N, 34(1): 159-182,2000$. MR1735971 (2000k:65203)

[5] R. Beck, R. Hiptmair, and B. Wohlmuth. Hierarchical error estimator for eddy current computation. In ENUMATH99: Proceedings of the 3rd European Conference on Numerical Mathematics and Advanced Applictions (ed. P. Neitaanmäki and T. Tiihonen), 110-120. World Scientific, Singapore, 2000. MR 1936173

[6] D. Boffi. Discrete compactness and Fortin operator for edge elements. Numer. Math., 87:229 246, 2000. MR1804657 (2001k:65168)

[7] D. Boffi. A note on the discrete compactness property and the de Rham diagram. Appl. Math. Letters, 14:33-38, 2001. MR1793699 (2001g:65145)

[8] A. Bossavit. Mixed finite elements and the complex of Whitney forms. In J. Whiteman, editor, The Mathematics of Finite Elements and Applications VI, 137-144. Academic Press, London, 1988. MR $956893(89 \mathrm{k}: 58028)$

[9] D. Braess and J. Schöberl. Equilibrated residual error estimators for Maxwell's equations. Technical Report 2006-19, Johann Radon Institute for Computational and Applied Mathematics (RICAM), 2006.

[10] C. Carstensen. A posteriori error estimate for the mixed finite element method. Math. Comp., 66:465-476, 1997. MR 1408371 (98a:65162)

[11] P. Clément. Approximation by finite element functions using local regularization. R.A.I.R.O. Anal. Numer., R2:77-84, 1975. MR0400739(53:4569)

[12] S. Cochez and S. Nicaise. Uniform a posteriori error estimation for the heterogeneous Maxwell equations. Report LAMAV 06.05, Université de Valenciennes et du Hainaut Cambrésis.

[13] L. Demkowicz and I. Babuška. Optimal $p$ interpolation error estimates for edge finite elements of variable order in 2d. Technical Report 01-11, TICAM, University of Texas at Austin, 2001.

[14] L. Demkowicz, P. Monk, L. Vardapetyan, and W. Rachowicz. De Rham diagram for $h p$ finite element spaces. Comput. Math. Appl., 39(7-8):29-38, 2000. MR1746160 (2000m:78052)

[15] V. Girault and P. A. Raviart. Finite Element Methods for Navier-Stokes Equations. Springer, Berlin, Heidelberg, New York, 1986. MR851383(88b:65129)

[16] R. Hiptmair. Multigrid method for Maxwell's equations. SIAM J. Numer. Anal., 36:204-225, 1999. MR 1654571 (99j:65229)

[17] P. Monk. A posteriori error indicators for Maxwell's Equations. J. Comp. Appl. Math., 100:173-190, 1998. MR.1659117(2000k:78020)

[18] P. Monk, Finite Element Methods for Maxwell's Equations. Oxford University Press, 2003. MR2059447 (2005d:65003) 
[19] J.-C. Nedelec. Mixed finite elements in $\mathbb{R}^{3}$. Numer. Math., 35:315-341, 1980. MR592160 (81k:65125)

[20] J.-C. Nédélec. A new family of mixed finite elements in $\mathbb{R}^{3}$. Numer. Math., 35:315-341, 1980. MR864305 (88e:65145)

[21] S. Nicaise and E. Creusé. A posteriori error estimation for the heterogeneous Maxwell equations on isotropic and anisotropic meshes. CALCOLO, 40:249-271, 2003. MR2025712 (2004j:65133)

[22] J. Pasciak and J. Zhao. Overlapping Schwarz methods in $\mathrm{H}(\mathrm{curl})$ on nonconvex domains. East West J. Numer. Anal., 10:221-234, 2002. MR1935967 (2003j:65108)

[23] P.-A. Raviart and J.-M. Thomas. A mixed finite element method for second order elliptic problems. In I. Galligani and E. Magenes, editors, Mathematical Aspects of the Finite Element Method, Lecture Notes in Mathematics, pages 292-315. Springer, Berlin, 1977. MR 0483555 (58:3547)

[24] L. R. Scott and S. Zhang. Finite element interpolation of nonsmooth functions satisfying boundary conditions. Math. Comp., 54(190):483-493, 1990. MR1011446 (90j:65021)

[25] J. Schöberl. Commuting quasi-interpolation operators for mixed finite elements. Report ISC-01-10-MATH, Texas A\&M University, available from www.isc.tamu.edu/iscpubs/iscreports.html, 2001.

[26] J. Schöberl and S. Zaglmayr. High order Nédélec elements with local complete sequence properties. Int. J. for Computation and Maths. in Electrical and Electronic Eng. COMPEL, to appear, 2005. MR2169504

[27] J. Schöberl. A multilevel decomposition result in $H$ (curl). Proceedings of the $8^{\text {th }}$ European Multigrid Conference EMG 2005, TU Delft (to appear).

[28] A. Toselli. Overlapping Schwarz methods for Maxwell's equations in three dimension. Numer. Math., 86:733-752, 2000. MR 1794350 (2001h:65137)

[29] A. Toselli and O. Widlund. Domain Decomposition Methods - Algorithms and Theory. Springer Series in Computational Mathematics, Vol. 34, 2005. MR2104179 (2005g:65006)

[30] R. Verfürth. A Review of A Posteriori Error Estimation and Adaptive Mesh-Refinement Techniques. Wiley-Teubner, 1996.

[31] J. P. Webb, Hierarchal vector basis functions of arbitrary order for triangular and tetrahedral finite elements, IEEE Trans. on Antennas and Propagation, 47:1244-1253, 1999. MR.1711458 (2000g:78031)

[32] Sabine Zaglmayr, High Order Finite Element Methods for Electromagnetic Field Computation. Ph.D. thesis, Institute for Computational Mathematics, Johannes Kepler University Linz, 2006

Center for Computational Engineering Science, RWTH Aachen University, PauwelStrasse 19, D-52074 Aachen, Germany

URL: http://www.hpfem.jku.at/people/joachim 